\title{
BLIND FRAME SYNCHRONIZATION AND PHASE OFFSET ESTIMATION FOR CODED SYSTEMS
}

\author{
Rodrigue Imad and Sebastien Houcke
}

\author{
TELECOM Bretagne \\ Technopole Brest Iroise - CS 83818 - 29238 Brest Cedex 3, France \\ phone: + (33)229001381, fax: + (33)229001012, email: \{firstname\}.\{lastname\}@ telecom-bretagne.eu
}

\begin{abstract}
In this paper, we present a new algorithm of blind frame synchronization and phase offset estimation that can be applied to any digital transmission scheme using a channel coding with a Binary Phase Shift Keying (BPSK) modulation. The estimator is based on the calculation of the syndrome elements of a received codeword obtained using the parity check matrix of the code. After presenting the proposed method, we evaluate its performance by applying it to some Low Density Parity Check (LDPC) codes and convolutional codes. This performance is measured by plotting the probability of false frame synchronization and the Mean Squared Estimation Error (MSEE) versus the Signal to Noise Ratio $\left(E_{b} / N_{0}\right)$.
\end{abstract}

\section{INTRODUCTION}

Nowadays, the transmission and storage of information are based on digital techniques. An important step in these techniques is Channel Coding. Several studies [1,2] have demonstrated that actual codes such as LDPC codes and turbocodes, are very powerful and are able to decode heavily corrupted sequences. However, these works were based on the assumption of a coherent detection, which may be less than realistic for many digital communication systems. Therefore, we are interested in deriving new algorithms that are able to estimate the frame synchronization position and the phase offset introduced by the propagation channel, before applying the decoder.

In the literature, many algorithms have been proposed for the estimation of the phase offset introduced by the channel. For a phase offset constant on a whole received codeword and by assuming that we do not have any a priori information about the transmitted data, two synchronization approaches are often used: the "Non Data Aided" (NDA) and the "Hard Decision Directed" (HDD) [3]. These approaches assume that only the modulation type used before transmission is known by the receiver, which is generally the case. In addition, some algorithms of phase offset estimation for coded signals considered the "Code aided" hypothesis, such as recently in [4], while others considered the phase recovery jointly with the decoder [5].

We present in this paper a new method of blind phase recovery based on the calculation of the syndrome elements of a received sequence of coded symbols. This method has been inspired from a blind frame synchronization method that has been introduced and developed in $[6,7,8]$.

This paper is organized as follows. In Section 2 we define and introduce the context of our study. A review on the frame synchronization method recently proposed in the case of perfect phase recovery, is presented in Section 3. In Section 4 we modify the previous method to take into consideration the phase offset due to the channel. Section 5 introduces the proposed method for estimating the phase offset. Simulation results are presented in Section 6 where different LDPC and convolutional codes are synchronized. Finally, Section 7 concludes the work.

\section{CONTEXT OF OUR STUDY}

In this paper, we consider that the transmitter is sending a binary sequence of codewords and is using a Binary Phase Shift Keying (BPSK) modulation. The propagation channel is corrupted by an additive white Gaussian noise.

For a given code of rate

$$
\rho=\frac{n_{c}-n_{r}}{n_{c}}
$$

it is often possible to find the corresponding parity check matrix $H$ of size $n_{r} \times n_{c}$, where $n_{c}$ represents the length of a codeword and $n_{r}$ the number of parity relations. Let $b(k)=$ \pm 1 be the $k^{t h}$ coded and modulated bit to be transmitted. At the reception, the $k^{\text {th }}$ received sample is given by:

$$
r(k)=b\left(k-t_{0}\right) e^{i \theta}+w(k),
$$

where $t_{0}$ is an integer representing the shift of the transmitted symbols and $\theta$ is a real representing the phase offset introduced by the propagation channel. $w(k)=w_{1}(k)+i w_{2}(k)$ is a white complex Gaussian noise. The received sequence of $N$ samples can be written as:

$$
r=[r(1), \ldots, r(N)] .
$$




\section{FRAME SYNCHRONIZATION METHOD IN THE CASE OF PERFECT PHASE RECOVERY}

When there is no phase offset introduced by the propagation channel, the phase $\theta$ introduced in (1) is equal to zero.

We showed in [8] that our blind frame synchronization method is based on a MAP approach in the sense of maximizing the probability that a position $t$ corresponds to the correct synchronization moment, given the received samples. In other words, it maximizes the following a posteriori probability:

$$
\operatorname{Pr}[t / r], t \in\left[0, n_{c}-1\right] .
$$

Therefore, at each position $t$ on the received sequence we apply a sliding window of length $K n_{c}$, where $K$ is an integer greater or equal to one. $K$ represents the number of blocks of length $n_{c}$ contained in the synchronization window.

At each position of this sliding window, we calculate the vector of Log-Likelihood Ratios (LLR) of the syndrome elements. This vector can be written as:

$$
L\left(S_{t}\right)=\left[L\left(S_{t}(1)\right), \ldots, L\left(S_{t}\left(K n_{r}\right)\right)\right],
$$

where $L\left(S_{t}(k)\right)$ is the LLR of the $k^{t h}$ syndrome element. According to [9], $L\left(S_{t}(k)\right)$ is proportional to:

$$
\hat{L}\left(S_{t}(k)\right)=(-1)^{u_{k}+1} \prod_{j=1}^{u_{k}} \operatorname{sign}\left(r\left(t+k_{j}\right)\right) \min _{j=1, \ldots, u_{k}}\left|r\left(t+k_{j}\right)\right|,
$$

where $u_{k}$ and $k_{j}$ represent the number of ones in the $k^{t h}$ line of the parity check matrix of the code and the position of the $j^{t h}$ non zero element in this $k^{\text {th }}$ line, respectively. Having this, we compute

$$
\hat{\phi}(t)=\sum_{k=1}^{K n_{r}} \hat{L}\left(S_{t}(k)\right)
$$

and the frame synchronization position is estimated by:

$$
\hat{t}_{0}=\underset{t=0, \ldots, n_{c}-1}{\operatorname{argmin}}\{\hat{\phi}(t)\} .
$$

\section{FRAME SYNCHRONIZATION IN THE CASE OF A PHASE OFFSET INTRODUCED BY THE CHANNEL}

Let us study the robustness of our frame synchronization method when a phase offset $\theta$ is introduced by the propagation channel and assumed to be constant on $K$ received codewords.

In order to calculate the reliability of the sum of the syndrome elements, we apply our frame synchronization criterion twice; once on the real part of a received symbol $r(k)$ and the other time on the imaginary part. This gives two functions:

$$
\begin{gathered}
P_{\theta}(t)=\sum_{k=1}^{K n_{r}}(-1)^{u_{k}+1} \prod_{j=1}^{u_{k}} \operatorname{sign}\left(\Re\left(r\left(t+k_{j}\right)\right)\right) \\
\min _{j=1, \ldots, u_{k}}\left|\Re\left(r\left(t+k_{j}\right)\right)\right| \\
=\sum_{k=1}^{K n_{r}}(-1)^{u_{k}+1} \prod_{j=1}^{u_{k}} \operatorname{sign}\left(b\left(t+k_{j}-t_{0}\right) \cos \theta+w_{1}\left(t+k_{j}\right)\right) \\
\min _{j=1, \ldots, u_{k}}\left|b\left(t+k_{j}-t_{0}\right) \cos \theta+w_{1}\left(t+k_{j}\right)\right|
\end{gathered}
$$

and

$$
\begin{gathered}
Q_{\theta}(t)=\sum_{k=1}^{K n_{r}}(-1)^{u_{k}+1} \prod_{j=1}^{u_{k}} \operatorname{sign}\left(\Im\left(r\left(t+k_{j}\right)\right)\right) \\
\min _{j=1, \ldots, u_{k}}\left|\Im\left(r\left(t+k_{j}\right)\right)\right| \\
=\sum_{k=1}^{K n_{r}}(-1)^{u_{k}+1} \prod_{j=1}^{u_{k}} \operatorname{sign}\left(b\left(t+k_{j}-t_{0}\right) \sin \theta+w_{2}\left(t+k_{j}\right)\right) \\
\min _{j=1, \ldots, u_{k}}\left|b\left(t+k_{j}-t_{0}\right) \sin \theta+w_{2}\left(t+k_{j}\right)\right| .
\end{gathered}
$$

Let $E[x]$ denote the expected value of $x$. We have for example:

$$
E\left[\lim _{\theta \rightarrow 0} P_{\theta}(t)\right]=E[\hat{\phi}(t)] \text { and } E\left[\lim _{\theta \rightarrow 0} Q_{\theta}(t)\right]=0
$$

and on the other hand

$$
E\left[\lim _{\theta \rightarrow \frac{\pi}{2}} P_{\theta}(t)\right]=0 \text { and } E\left[\lim _{\theta \rightarrow \frac{\pi}{2}} Q_{\theta}(t)\right]=E[\hat{\phi}(t)]
$$

$\theta$ being unknown, $P_{\theta}(t)$ and $Q_{\theta}(t)$ should then be treated together in order to get an idea on the reliability of the syndrome elements. Therefore, an estimation of the frame synchronization position is obtained similarly to (2) but by replacing $\hat{\phi}(t)$ by $F\left(P_{\theta}(t), Q_{\theta}(t)\right)$, which is a function of $P_{\theta}(t)$ and $Q_{\theta}(t)$. The choice of $F$ depends on the fact that $u_{k}$ is even or odd.

\section{Case $\mathbf{I}: u_{k}$ is even}

In a noise free channel, (3) and (4) can be written as:

$$
\begin{aligned}
P_{\theta}(t) & =\hat{\phi}(t)|\cos \theta| \\
Q_{\theta}(t) & =\hat{\phi}(t)|\sin \theta|
\end{aligned}
$$

In the case of a coherent detection, we usually have $\hat{\phi}\left(t_{0}\right)<$ $0<\hat{\phi}(t)_{t \neq t_{0}}$. Therefore, in order to apply the criterion given in (2) to our case, $F(P, Q)$ should be an increasing function of $P$ and $Q$. A simple example of $F$ giving good results could be:

$$
F\left(P_{\theta}(t), Q_{\theta}(t)\right)=P_{\theta}(t)+Q_{\theta}(t) .
$$




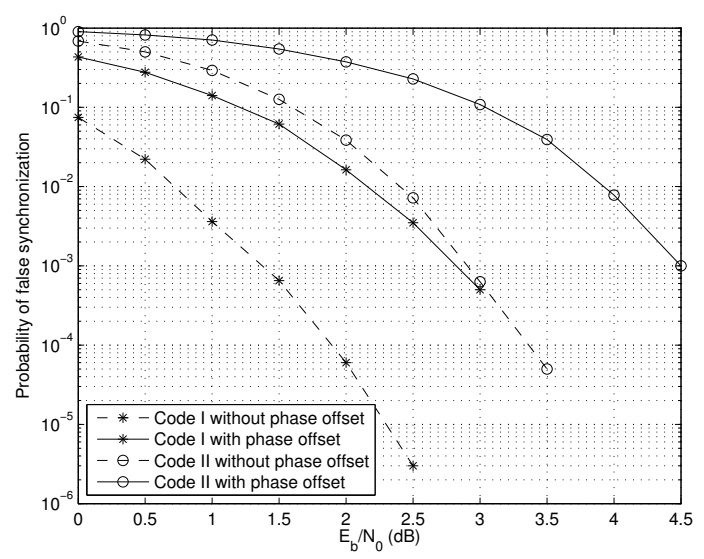

Fig. 1. Frame synchronization of LDPC codes when a random phase offset is introduced.

\section{Case II: $u_{k}$ is odd}

When $u_{k}$ is odd and in a noise free channel, we have:

$$
\begin{gathered}
P_{\theta}(t)=\left\{\begin{aligned}
\hat{\phi}(t)|\cos \theta| & \text { if } \theta \in\left[0, \frac{\pi}{2}\right] \cup\left[\frac{3 \pi}{2}, \pi\right] \\
-\hat{\phi}(t)|\cos \theta| & \text { if } \theta \in\left[\frac{\pi}{2}, \frac{3 \pi}{2}\right]
\end{aligned}\right. \\
Q_{\theta}(t)=\left\{\begin{aligned}
\hat{\phi}(t)|\sin \theta| & \text { if } \theta \in[0, \pi] \\
-\hat{\phi}(t)|\sin \theta| & \text { if } \theta \in[\pi, 2 \pi]
\end{aligned}\right.
\end{gathered}
$$

The sign of $P_{\theta}(t)$ and $Q_{\theta}(t)$ being dependent from $\theta$, it does not help us this time for finding the frame synchronization position. However we still have:

$$
\begin{aligned}
& E\left[\left|P_{\theta}\left(t_{0}\right)\right|\right]>E\left[\left|P_{\theta}(t)_{t \neq t_{0}}\right|\right] \\
& \text { and } \quad E\left[\left|Q_{\theta}\left(t_{0}\right)\right|\right]>E\left[\left|Q_{\theta}(t)_{t \neq t_{0}}\right|\right]
\end{aligned}
$$

According to the previous observations, the function given in (7) becomes:

$$
F\left(P_{\theta}(t), Q_{\theta}(t)\right)=-\left|P_{\theta}(t)\right|-\left|Q_{\theta}(t)\right|
$$

\section{PROPOSED METHOD OF CARRIER PHASE RECOVERY}

Let us assume that we have correctly estimated the frame synchronization position. In a noise free channel and by combining (5), (6), (8) and (9) we get:

$$
\theta=\operatorname{Arctan}\left( \pm \frac{Q_{\theta}\left(t_{0}\right)}{P_{\theta}\left(t_{0}\right)}\right) \quad \forall u_{k}
$$

Using the above equation in our context, we get an estimation of $\theta$ with an ambiguity of $\frac{\pi}{2}$. However, we can evolve this criterion in order to obtain an estimation of $\theta$ modulo $\pi$ instead of $\frac{\pi}{2}$. The procedure to be done depends on the parity of $u_{k}$ : If $u_{k}$ is even, we study the evolution of the functions $\delta x \mapsto$

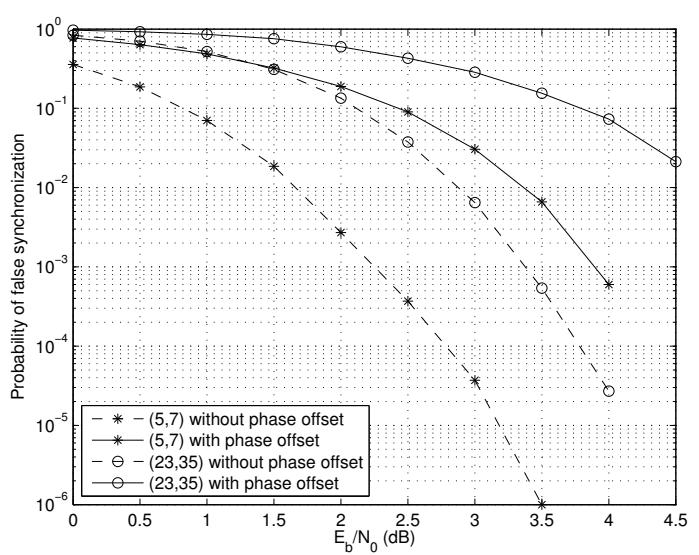

Fig. 2. Frame synchronization of convolutional codes when a random phase offset is introduced.

$P_{\theta+\delta x}$ and $\delta x \mapsto Q_{\theta+\delta x}$. Indeed, for $\delta x$ positive and close to zero, and as $P_{\theta}\left(t_{0}\right)$ and $Q_{\theta}\left(t_{0}\right)$ are negative and depend on $\theta$, we have:

- If $\theta \in\left[0, \frac{\pi}{2}-\delta x\right] \cup\left[\pi, \frac{3 \pi}{2}-\delta x\right], \delta x \mapsto P_{\theta+\delta x}$ is an increasing function and $\delta x \mapsto Q_{\theta+\delta x}$ is a decreasing one.

- If $\theta \in\left[\frac{\pi}{2}, \pi-\delta x\right] \cup\left[\frac{3 \pi}{2}, 2 \pi-\delta x\right], \delta x \mapsto P_{\theta+\delta x}$ is a decreasing function and $\delta x \mapsto Q_{\theta+\delta x}$ is an increasing one.

However, when $u_{k}$ is odd, we can use the same procedure as the one described above but by studying this time the variations of $\delta x \mapsto\left|P_{\theta+\delta x}\right|$ and $\delta x \mapsto\left|Q_{\theta+\delta x}\right|$. We have:

- If $\theta \in\left[0, \frac{\pi}{2}-\delta x\right] \cup\left[\pi, \frac{3 \pi}{2}-\delta x\right], \delta x \mapsto\left|P_{\theta+\delta x}\right|$ is a decreasing function and $\delta x \mapsto\left|Q_{\theta+\delta x}\right|$ an increasing one.

- If $\theta \in\left[\frac{\pi}{2}, \pi-\delta x\right] \cup\left[\frac{3 \pi}{2}, 2 \pi-\delta x\right], \delta x \mapsto\left|P_{\theta+\delta x}\right|$ is an increasing function and $\delta x \mapsto\left|Q_{\theta+\delta x}\right|$ a decreasing one.

By applying this method, we are able to estimate $\theta$ with an ambiguity of $\pi$.

\section{SIMULATION RESULTS}

We present in this section simulation results concerning frame synchronization and phase offset estimation. The results below were obtained for $K=1$, which means that only one block is contained in the synchronization window. For all the plotted curves, functions given in (7) and (11) were used. In order to evaluate the performance of the proposed method, we synchronized different types of codes. For each simulation, more than 10000 Monte Carlo realizations were made. 


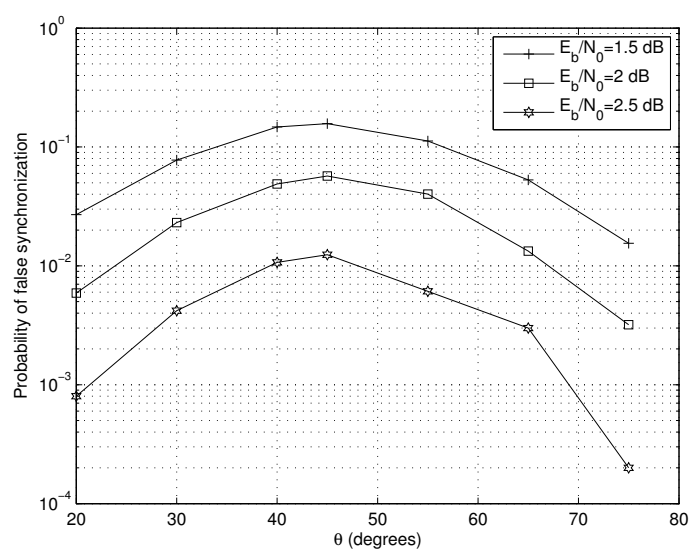

Fig. 3. Frame synchronization of LDPC code I versus $\theta$, for different values of $E_{b} / N_{0}$.

Fig.1 shows the curves of the probability of false frame synchronization versus $E_{b} / N_{0}$ for two LDPC codes. These codes have a length equals to 512 bits and a rate of 0.5 . They differ only by the composition of their parity check matrix. Code I has four non zero elements in each line of its parity check matrix and code II has six. When a phase offset randomly chosen by a uniform law between 0 and $2 \pi$ is introduced, there is a gap of around $1.5 \mathrm{~dB}$ compared to the case where no phase offset exists. Let us now compare the curves plotted for codes I and II: as we can see and as mentioned in [6, 7], there is a degradation in the performance of our synchronization method when the number of non zero elements in the parity check matrix of the code increases.

In Fig. 2, we applied the same synchronization method but this time to convolutional codes. We considered in our simulations that the final state of the encoder is not imposed. This means that neither we terminated the trellis nor we applied the "tail-biting" technique [10]. In these conditions, non zero elements in the parity check matrix of the code are placed in a "staircase" form. This leads to a degradation in the performance of our synchronization method. One simple solution to this problem is given in [7] where the authors propose to interleave the bits in each transmitted codeword using a pseudorandom interleaver of size $n_{c}$ bits.

Two convolutional codes of generator polynomials $(5,7)$ and $(23,35)$ were tested. They have same length (512 bits), same rate (0.5) and constraint lengths equal to 3 and 5 , respectively. As shown in Fig. 2, we observe the same behavior as the one obtained with LDPC codes.

Note that the number of non zero elements in each line of the parity check matrix of codes $(5,7)$ and $(23,35)$ is equal to 5 and 7 , respectively. Thus, even if $u_{k}$ is odd, the proposed frame synchronization method presents good performance.

Let us now apply the frame synchronization procedure for a given $\theta$. Figure 3 shows the performance of the frame syn-

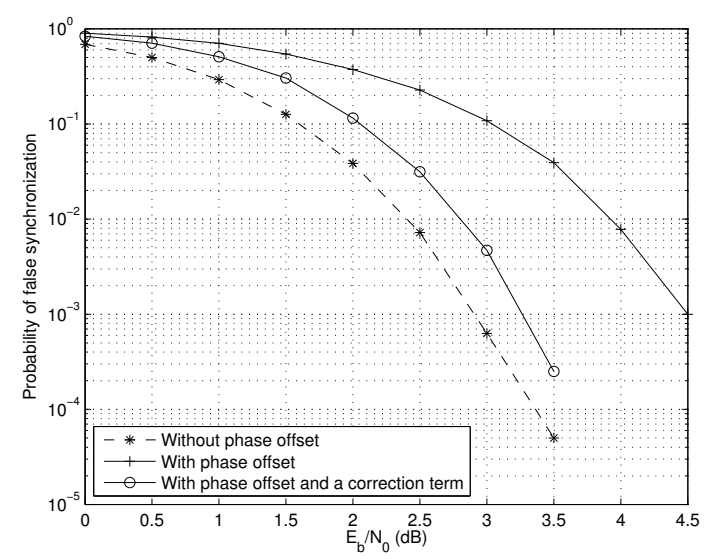

Fig. 4. Frame synchronization of LDPC code II by adding a correction term to the received samples.

chronization method applied to LDPC code I, previously introduced in this section. As we can see in this figure, each plotted curve represents the probability of false synchronization versus $\theta$, for a different $E_{b} / N_{0}$. We can clearly observe that the frame synchronization performance varies in terms of the phase offset of the channel. For $\theta=\frac{\pi}{4}$, the performance of our method is the worse.

Having this, a correction term $\theta_{c}$ can be added to the received samples in order to improve the frame synchronization performance. Therefore, let $\theta_{c}(i)_{i=1, \ldots, 4}=\frac{\pi}{16}, \frac{\pi}{8}, \frac{3 \pi}{16}$ and $\frac{\pi}{4}$, respectively. Each time we correct a received block by $\theta_{c}(i)_{i=1, \ldots, 4}$, then we apply our frame synchronization procedure, we get $F\left(P_{\theta+\theta_{c}(i)}(t), Q_{\theta+\theta_{c}(i)}(t)\right)$. The frame synchronization position is finally estimated by:

$$
\hat{t}_{0}=\underset{\substack{t=0, \ldots, n_{c}-1 \\ \theta_{c}(i)=\theta_{c}(1), \ldots, \theta_{c}(4)}}{\operatorname{argmin}}\left(F\left(P_{\theta+\theta_{c}(i)}(t), Q_{\theta+\theta_{c}(i)}(t)\right)\right) .
$$

Fig. 4 shows the performance of the above procedure once applied to LDPC code II. At a probability of false synchronization equals to $10^{-3}$, an improvement of around $1.2 \mathrm{~dB}$ is observed.

Let us now evaluate the robustness of the method proposed in Section 5 concerning the phase offset estimation, by comparing its performance to the HDD algorithm. Being used as a reference for many phase recovery algorithms, the HDD algorithm is based on the estimation of the transmitted symbols by taking hard decisions on the received signal. The estimated symbols are then introduced in the expression of the phase likelihood and an estimation of the phase offset is given by:

$$
\hat{\phi}_{H D D}=\arg \left(\sum_{k=1}^{N} r(k) \hat{d}(k)^{*}\right),
$$

where $\{\hat{d}(k)\}_{k=1, \ldots, N}$ are the hard decision estimations for $N$ transmitted symbols obtained from the received samples 


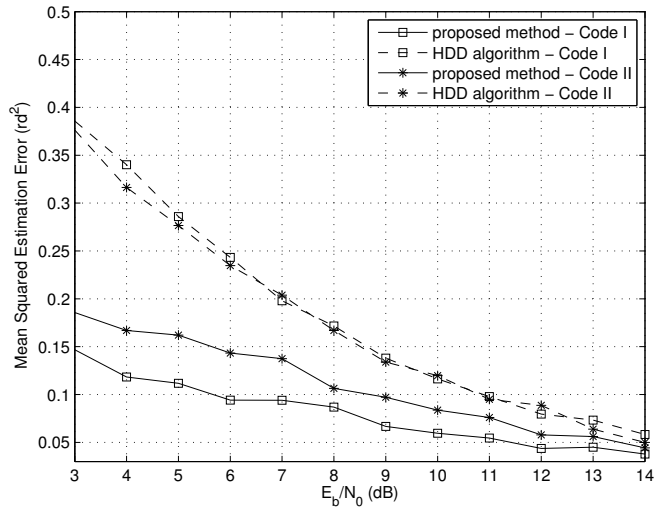

Fig. 5. Comparison between the proposed method and the HDD method concerning the phase offset estimation for LDPC codes.

$\{r(k)\}_{k=1, \ldots, N} \cdot\left(^{*}\right)$ designates the conjugate of a complex number.

We assume that the frame synchronization position has been correctly estimated. Fig. 5 shows the mean squared estimation error (in $r d^{2}$ ) versus $E_{b} / N_{0}$, once our proposed phase recovery method is applied to LDPC codes I and II previously introduced in this section. Compared to the HDD approach, our method presents better performance. A large gap between the two methods can be clearly seen especially for low $E_{b} / N_{0}$. In Fig. 6, same curves have been plotted for convolutional codes $(5,7)$ and $(23,35)$. Our proposed phase recovery method is always better than the HDD algorithm.

\section{CONCLUSION}

We have proposed in this paper a new method of blind synchronization that exploits the redundancy introduced by error correcting codes. The criterion on which our method is based on, is used for the frame synchronization and the phase offset estimation at the same time. As shown by simulations, the frame synchronization method keeps good performance when an arbitrary phase offset is added to the transmitted symbols. Furthermore, we have shown that compared to the HDD algorithm, our method of estimating the phase offset presents better performance.

\section{REFERENCES}

[1] C. Berrou, A. Glavieux, and P. Thitimajshima, "Near shannon limit error-correcting coding and decoding: turbo-codes," ICC, pp. 1067-1070, May 1993.

[2] D. J. C. MacKay and R. M. Neal, "Near shannon-limit

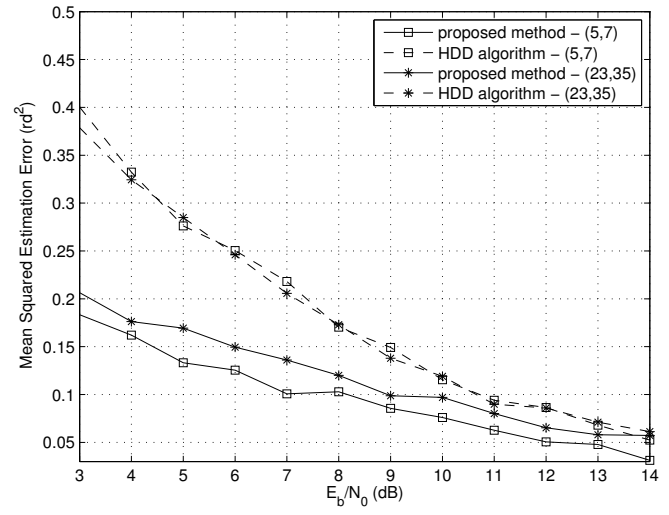

Fig. 6. Comparison between the proposed method and the HDD method concerning the phase offset estimation for convolutional codes.

performance of low-density parity-check codes," Electronics Letters, vol. 32, pp. 1645-1646, August 1996.

[3] H. Meyr, M. Moeneclaey, and S. A. Fechtel, Digital Communication Receivers. Synchronization, Channel Estimation and Signal Processing. J. Wiley and Sons, 1998.

[4] H. Wymeersch, H. Steendam, H. Bruneel, and M. Moeneclaey, "Code-aided frame synchronization and phase ambiguity resolution," IEEE Transactions on Signals Processing, vol. 54, pp. 2747-2757, July 2006.

[5] B. Mielczarek and A. Svensson, "Joint synchronization and decoding of turbo codes on AWGN channels," VTC, vol. 3, pp. 1886-1890, May 1999.

[6] S. Houcke and G. Sicot, "Blind frame synchronization for block code," in Proc. of EUSIPCO, European Sig. Proc., September 2006.

[7] R. Imad, S. Houcke, and C. Douillard, "Blind frame synchronization on gaussian channel," in Proc. of EUSIPCO, European Sig. Proc., September 2007.

[8] R. Imad, G. Sicot, and S. Houcke, "Blind frame synchronization for error correcting codes," submitted to IEEE Trans. Comm.

[9] J. Hagenauer, E. Offer, and L. Papke, "Iterative decoding of binary block and convolutional codes," IEEE trans. on Information Theory, vol. 42, no. 2, pp. 429445, March 1996.

[10] R. Johannesson and K. Sh. Zigangirov, Fundamentals of Convolutional Coding, ser. IEEE series on Digital and Mobile Communication. IEEE Press, 1999. 